\title{
Whirlin, a cytoskeletal scaffolding protein, stabilizes the paranodal region and axonal cytoskeleton in myelinated axons
}

\author{
James A Green ${ }^{1}$, Jun Yang ${ }^{2}$, M'hamed Grati ${ }^{3}$, Bechara Kachar ${ }^{3}$ and Manzoor A Bhat ${ }^{1,4^{*}}$
}

\begin{abstract}
Background: Myelinated axons are organized into distinct subcellular and molecular regions. Without proper organization, electrical nerve conduction is delayed, resulting in detrimental physiological outcomes. One such region is the paranode where axo-glial septate junctions act as a molecular fence to separate the sodium $\left(\mathrm{Na}^{+}\right)$ channel-enriched node from the potassium $\left(\mathrm{K}^{+}\right)$channel-enriched juxtaparanode. A significant lack of knowledge remains as to cytoskeletal proteins which stabilize paranodal domains and underlying cytoskeleton. Whirlin (Whrn) is a PDZ domain-containing cytoskeletal scaffold whose absence in humans results in Usher Syndromes or variable deafness-blindness syndromes. Mutant Whirlin (Whrn) mouse model studies have linked such behavioral deficits to improper localization of critical transmembrane protein complexes in the ear and eye. Until now, no reports exist about the function of Whrn in myelinated axons.

Results: RT-PCR and immunoblot analyses revealed expression of Whrn mRNA and Whrn full-length protein, respectively, in several stages of central and peripheral nervous system development. Comparing wild-type mice to Whrn knockout (Whrn ${ }^{-1-}$ ) mice, we observed no significant differences in the expression of standard axonal domain markers by immunoblot analysis but observed and quantified a novel paranodal compaction phenotype in 4 to 8 week-old $\mathrm{Whrn}^{-1-}$ nerves. The paranodal compaction phenotype and associated cytoskeletal disruption was observed in $\mathrm{Whrn}^{-1-}$ mutant sciatic nerves and spinal cord fibers from early (2 week-old) to late (1 year-old) stages of development. Light and electron microscopic analyses of Whrn knockout mice reveal bead-like swellings in cerebellar Purkinje axons containing mitochondria and vesicles by both. These data suggest that Whrn plays a role in proper cytoskeletal organization in myelinated axons.

Conclusions: Domain organization in myelinated axons remains a complex developmental process. Here we demonstrate that loss of Whrn disrupts proper axonal domain organization. Whrn likely contributes to the stabilization of paranodal myelin loops and axonal cytoskeleton through yet unconfirmed cytoskeletal proteins. Paranodal abnormalities are consistently observed throughout development ( $2 \mathrm{wk}-1 \mathrm{yr}$ ) and similar between central and peripheral nervous systems. In conclusion, our observations suggest that Whrn is not required for the organization of axonal domains, but once organized, Whrn acts as a cytoskeletal linker to ensure proper paranodal compaction and stabilization of the axonal cytoskeleton in myelinated axons.
\end{abstract}

Keywords: Myelinated axons, Axonal domains, Paranodal domain, Axonal cytoskeleton, Whirlin

\footnotetext{
* Correspondence: bhatm@uthscsa.edu

${ }^{1}$ Department of Cell and Molecular Physiology, University of North Carolina

School of Medicine, Chapel Hill, NC 27599, USA

${ }^{4}$ Department of Physiology, University of Texas School of Medicine, Health

Science Center, 7703 Floyd Curl Drive, San Antonio, TX 78229, USA

Full list of author information is available at the end of the article
} 


\section{Background}

Nervous system function depends on proper molecular organization between neurons and glial cells. In myelinated neurons, the segregation and enrichment of proteins in the defined domains, the Node of Ranvier, paranode, and juxtaparanode, is critical for saltatory action potential propagation [1-4]. Without proper organization, electrical nerve conduction is delayed and can result in significant motor and sensory deficits. The paranodal domain is a region of direct interaction between the glial myelin membrane loops and neuronal plasma membrane. Axoglial septate junctions (AGSJ) link these glial membrane loops to the axonal membrane and establish the paranodal ionic barrier separating sodium $\left(\mathrm{Na}^{+}\right)$channel-enriched node from the potassium $\left(\mathrm{K}^{+}\right)$channel-enriched juxtaparanode [5]. AGSJ's are composed of three transmembrane proteins: Contactin (Cont) [6], Contactin-associated protein (Caspr) [7], and Neurofascin155 (glial-derived $155 \mathrm{kDa}$ isoform) [8]. Genetic ablation of any of the three molecules disrupts the paranodal barrier function and results in degraded action potential propagation [6-11]. While much research has focused on these three proteins, other molecules contribute to domain formation and stabilization. For example, Caspr2 and TAG-1 are required for the organization of the juxtaparanodal domain and localization of potassium $\left(\mathrm{K}^{+}\right)$channels [12]. For long-term stability, Caspr and Caspr2 are thought to rely on cytoskeletal scaffold proteins to link their associated complexes with the axonal cytoskeleton. Loss of Caspr results in significant cytoskeletal disorder [9], and since its extracellular partner Cont lacks an intracellular c-terminus, the c-terminus of Caspr is likely a critical region of interaction between AGSJ's and the axonal cytoskeleton. Previous studies using $4.1 \mathrm{~B}$ mutant mice revealed that the $4.1 \mathrm{~B}$ cytoskeletal protein contributes to paranodal and juxtaparanodal stability $[13,14]$. A recent report also highlights $4.1 \mathrm{G}$ as an organizer of internodes in the peripheral nervous system [15]. The neuron is abundant with cytoskeletal scaffolds however, and such findings do not exclude the possibility that other cytoskeletal elements may contribute to paranodal maintenance and long-term stability.

Whrn is a cytoskeletal scaffold protein that plays an important role in vision and hearing [16,17]. In humans, WHIRLIN (WHRN) (DFNB31) mutations have been linked to Usher syndrome Type II (USH2), an autosomal recessive vision-hearing impairment disorder $[18,19]$. In mice, the Whrn coding sequence consists of 12 exons with two dominant splice variants, a full length and short (exon 5-12) isoform. Both variants contain a Proline-rich domain and PDZ-domain(s) which have been shown to link submembranous cytoskeletal elements to transmembrane complexes, as well as to self-oligomerize [20]. In the eye, Whrn interacts with the transmembrane proteins
Usherin (USH2) and Very Large G protein-coupled Receptor-1 (VLGR1/GPR98) within the periciliary membrane complex of photoreceptors. Likewise in the inner ear, Whrn interacts with Usherin and VLGR1/GPR98, which form the stereociliary ankle-links. Additionally, Whrn interacts indirectly with $4.1 \mathrm{~B}[21]$ and $4.1 \mathrm{R}$ through Mpp1/p55 [22], and directly with Myosin XVa [23]. Finally, Whrn is implicated with Esp8 in stereocilialength regulation [24]. While the function of Whrn in the ear and the eye has received significant attention, very little is known about its function in the central and/or peripheral nervous system. There are reports of Whrn protein expression in the cerebrum, cerebellum, and brainstem in wild-type mice and the protein is absent in Whrn knockout $\left(\mathrm{Whrn}^{-/-}\right)$and whirler $\left(\mathrm{Whrn}^{\text {wi/wi }}\right)$ mutant mice [25]. In Drosophila, the closest homolog to Whrn is dyschronic (dysc) [26]. In dysc mutants there is arrhythmic locomotor behavior but the eclosion circadian rhythms and clock protein cycling is unaffected [26]. Here we report that Whrn is involved in proper compaction of the paranodal region in myelinated axons and for proper stabilization of the axonal cytoskeleton.

\section{Results and discussion}

Whrn is expressed in central and peripheral nervous system tissues throughout development

Whrn is a cytoskeletal scaffolding protein which functions to link membrane protein complexes to the cytoskeleton within hair cell stereocilia of the ear and photoreceptors in the eye. To begin assessing its function in myelinated neurons, we obtained Whrn exon 1 knockout mice [17]. As reported previously, the murine Whrn locus consists of 12 exons with two dominant splice variants, a full length $(\sim 4 \mathrm{~kb})$ isoform and a short ( 2.5 kb) isoform (Figure 1A). Both variants contain PDZ-domains (Figure 1A, yellow box) and a Proline-rich domain (Figure 1A, blue box). After initial back crossing to C57BL6 mouse strain, we identified and confirmed the Whrn genotype using PCR methods. To begin characterizing mRNA expression of Whrn in the central (CNS) and peripheral nervous systems (PNS), we examined the relative expression of Whrn by reverse transcriptase polymerase chain reaction (RT-PCR) in dorsal root ganglia (DRG), sciatic nerves (SN), and spinal cord (SC) tissues (Figure 1B) in postnatal 21 dayold mice. With this subset of tissues we could delineate the origin of Whrn expression as SC tissue is a combination of glial and neuronal nuclei, DRG is predominantly neuronal, and SN is principally glial cytoplasm and nuclei. Since Whrn has two major isoforms, we designed specific primers to distinguish the full length isoform (Exons 1-4) alone and those common to the full length and short isoforms (Exons 9-10). No expression of Whrn isoforms was observed in $\mathrm{Whrn}^{-/-}$mice (Figure 1B). Robust expression of Whrn (Exons 1-4) mRNA was observed in DRG 


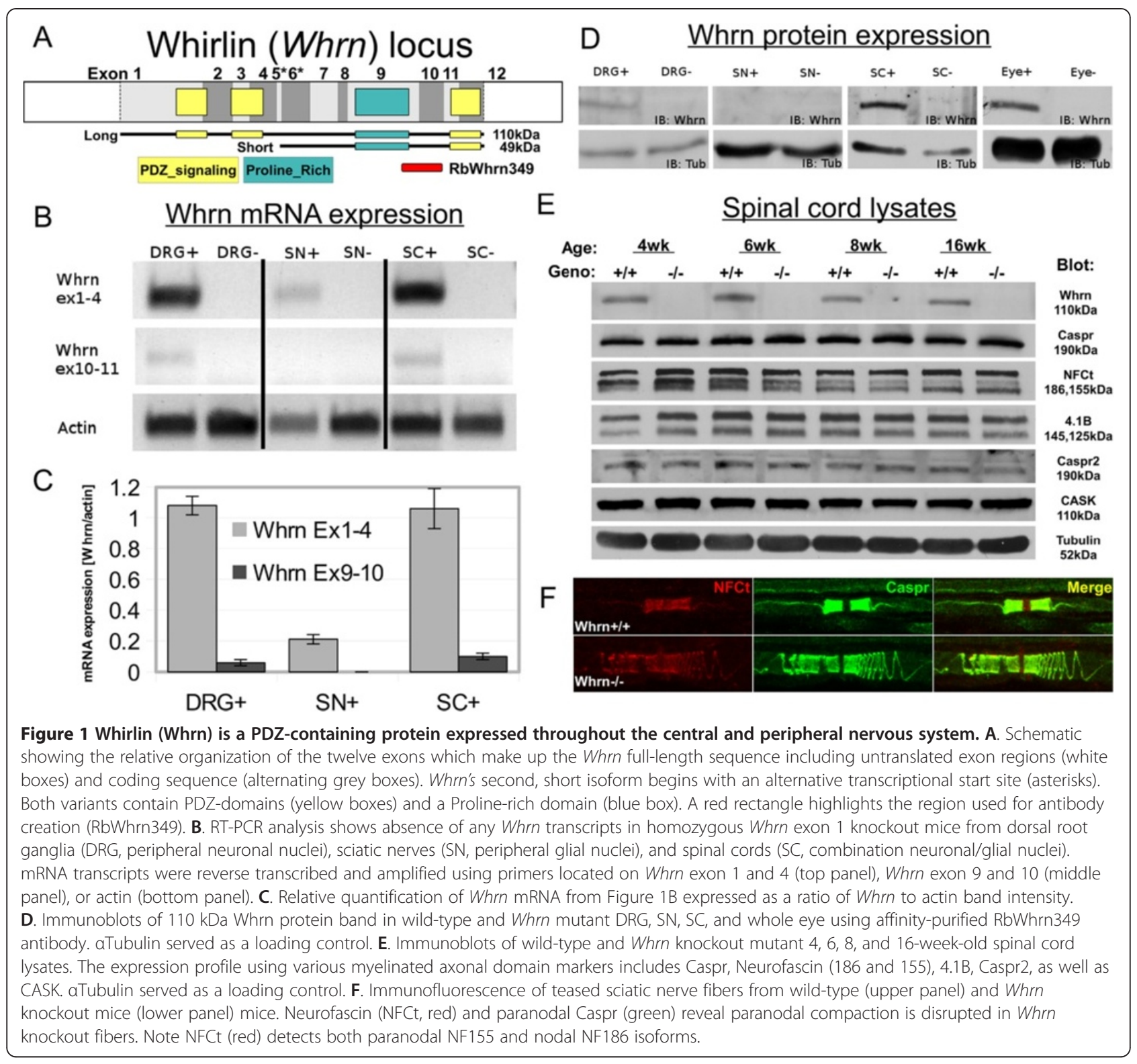

and SC tissue while weak expression was observed in $\mathrm{SN}$ tissue. Interestingly, weak expression of Whrn (Exons 9-10) mRNA was observed in DRG and SC tissue but no significant expression was observed in SN tissue. Actin (Exons 2-4) mRNA expression was used as a control for total RNA present. Relative expression was quantified as a ratio of Whrn to Actin mRNA between three total RT-PCR analyses (Figure 1C). After finding Whrn mRNA expression in the wild-type CNS and PNS neuronal tissues, we next sought to determine its protein expression in P30 $\mathrm{Whrn}^{+/+}$versus $\mathrm{Whrn}^{-/-}$mice by immunoblot analysis. In order to pursue these experiments, we generated several antibodies and affinitypurified one to the non-domain encoding, c-terminal region (aa699-804) of Whrn (Figure 1A, red bar). After affinity-purification, wild-type (+) and Whrn knockout (-) lysates derived from DRG, SN, SC, and whole eye were immunoblotted (Figure 1D, upper blots). Whole eye lysates were used as a positive control for protein expression based on previous reports [17]. A $110 \mathrm{kDa}$ Whrn band representing the full length protein was present in DRG, SC, and whole eye lysates. No $110 \mathrm{kDa}$ Whrn band was observed in $\mathrm{Whrn}^{-/-}$lysates or in wild-type SN lysate. Each tissue type showed similar total protein levels between genotypes based on total Tubulin on immunoblots (Figure 1D, lower blots). Having confirmed $\mathrm{Whrn}^{-1-}$ tissues were deficient in Whrn mRNA and protein, we next sought to determine if loss of Whrn resulted in altered protein expression of known nodal, paranodal, and juxtaparanodal proteins. We prepared spinal 
cord lysates from 4, 6, 8, and 16 week-old wild-type (+/+) and Whrn knockout (-/-) mice and immunoblotted for Whrn (110 kDa) (Figure 1E). Whrn protein expression levels were similar from 4 to 16 weeks in wild-type mice while no expression was observed in the $\mathrm{Whrn}^{-1-}$ at any time point. Next, we immunoblotted for the paranodal protein Caspr (190 kDa) and found similar expression levels between 4-16 week-old in both $\mathrm{Whrn}^{+/+}$and $\mathrm{Whrn}^{-/-}$mice. Using a pan-Neurofascin-c-terminal (NFct) antibody, we observed similar levels of nodal Neurofascin186 and paranodal Neurofascin155 protein expression in wild-type and Whrn knockout mice across 4-16 weeks of age. Examination of neuronal cytoskeletal protein $4.1 \mathrm{~B}$ and juxtaparanodal protein Caspr2 shows steady protein expression levels in wild-type and Whrn knockout mice from 4-16 weeks of age. Finally, previous reports showed in vivo interaction between rat Whrn/ CIP98 and calmodulin-dependent serine kinase (CASK) [27], a synaptic organization protein. After immunoblotting for CASK, no difference in its protein level was observed across Whrn genotype or ages from 4-16 weeks. Total protein levels in spinal cord lysates were consistent across the ages and genotypes based on Tubulin levels (Figure 1E). In summary, these developmental expression profiles suggest that loss of Whrn expression does not affect the overall expression or stability of other axonal domain markers.

\section{Whrn knockout mice reveal a quantifiable paranodal compaction phenotype in peripheral myelinated axons}

To determine the effects of Whrn loss on axonal domain organization, we examined well-characterized nodal, paranodal, and juxtaparanodal markers by immunofluorescence in wild-type and $\mathrm{Whrn}^{-/-}$mice. Extensive and repeated immunostaining with our Whrn antibody revealed no consistent localization or differences in Whrn localization between wild-type to $\mathrm{Whrn}^{-/-}$fibers (data not shown). Compared to wild-type, the most striking observation in teased $\mathrm{Whrn}^{-1-}$ sciatic nerve fibers was the spring-like separation (Figure 1F) of the paranodal axo-glial septate junction (AGSJ) loops beginning along the paranodaljuxtaparanodal border as observed by Caspr and NF155 immunostaining. Irregular paranodal compaction is rarely observed in wild-type fibers, so we sought to quantify the overall observation of this phenotype in $\mathrm{Whrn}^{+/+}$and $\mathrm{Whrn}^{-/-}$fibers. We utilized a blinded counting strategy to count spring-like phenotypes from wild-type $(\mathrm{N}=4676)$ or $\mathrm{Whrn}^{-/-}(\mathrm{N}=2798)$ Caspr-stained paranodes. A statistically significant difference (student t-test $\mathrm{p}=0.02$ ) was observed in the percentage of irregular paranodal compaction at $0.3 \%(\mathrm{SEM}=0.07 \%)$ in wild-type fibers compared to $1.8 \%(\mathrm{SEM}=0.45 \%)$ in Whrn knockout mice. Having confirmed the significance of this springlike paranodal phenotype in 7 week-old Whrn knockout fibers, we expanded our analysis to 4-8 week-old time points.

Sciatic nerves from $\mathrm{Whrn}^{+/+}$or $\mathrm{Whrn}^{-/-}$littermate mice at 4,6 , and 8 weeks, were immunostained with antibodies against nodal, paranodal, and juxtaparanodal markers (Figure 2). In the 4 week-old wild-type sciatic nerve (Figure 2Aa-d), juxtaparanodal $\mathrm{K}_{\mathrm{v}} 1.2$ (Figure 2Aa) was separated from nodal NF186 (Figure 2Ac) by paranodal Caspr (Figure 2Ab). This demarcation of domains (Figure 2Ad) in myelinated fibers is also observed in 4 week-old $\mathrm{Whrn}^{-/-}$fibers (Figure 2Bd). Myelin loops of AGSJs in 4 week-old $\mathrm{Whrn}^{-/-}$sciatic nerve fibers (Figure $2 \mathrm{Bb}$ ) were already separating away from the paranode compared to 4 week-old wild-type sciatic nerve fibers (Figure 2Ab). Breaks in $\mathrm{K}_{\mathrm{v}} 1.2$ localization (Figure 2Ba) were observed even where Caspr staining (Figure $2 \mathrm{Bb}$ ) was limited to a single myelin loop, suggesting AGSJ's barrier function was conserved. Juxtaparanodal localization was diffuse and asymmetric in $\mathrm{Whrn}^{-1-}$ fibers compared to wild-type (Figure 2Aa vs. 2Ba). When comparing wild-type to Whrn knockout mice, we observed no obvious differences in intensity levels of juxtaparanodal (Figure 2Aa vs. 2Ba) or nodal (Figure 2Ac vs. $2 \mathrm{Bc}$ ) markers. At 6 weeks, teased wild-type sciatic nerves (Figure 2Ca-d) showed typical domain organization. 6 week-old Whrn null sciatic nerves have typical NF186 (Figure 2Dc) nodal domain organization while Caspr paranodal compaction (Figure $2 \mathrm{Db}$ ) and $\mathrm{K}_{\mathrm{v}} 1.2$ juxtaparanodal (Figure 2Da) localization were disrupted. Additional immunostaining in 6 week-old Whrn null sciatic nerves with a pan-Neurofascin-C-terminal (NFct) antibody (Figure 2Ea) detected both nodal NF186 and paranodal NF155. Paranodal NF155 (Figure 2Ea, nonnodal signal) and Caspr (Figure 2Eb) immunostaining mostly colocalize (Figure 2Ed) in $\mathrm{Whrn}^{-/-}$sciatic nerve fibers and revealed similar problems with paranodal compaction. AnkG nodal (Figure 2Ec) domain organization in 6 week-old $\mathrm{Whrn}^{-/-}$sciatic nerve fibers appeared similar to wild-type (Figure 2Cc). Finally, immunostained 8 week-old sciatic nerves revealed similar NF186 nodal (Figure 2Fc vs. 2Gc) and AnkG nodal organization (Figure 2Hc). Like 4 and 6 week-old sciatic nerves, Caspr (Figure 2Gb, Hb) and NFct (Figure 2Ha) immunostaining consistently revealed paranodal compaction defects as well as $\mathrm{K}_{\mathrm{v}} 1.2$ juxtaparanodal (Figure 2Fa vs. 2Ga) diffusion in Whrn knockout mice when compared with wild-type (Figure 2Fb). To determine if such peripheral nerve phenotypes could be the result of differences in inner mesaxons, we immunostaining 7 week-old wild-type and Whrn knockout fibers with MAG but observed no striking phenotypic differences in localization (data not shown). In summary, the paranodal and juxtaparanodal regions displayed phenotypes that suggest that normal compaction of the 

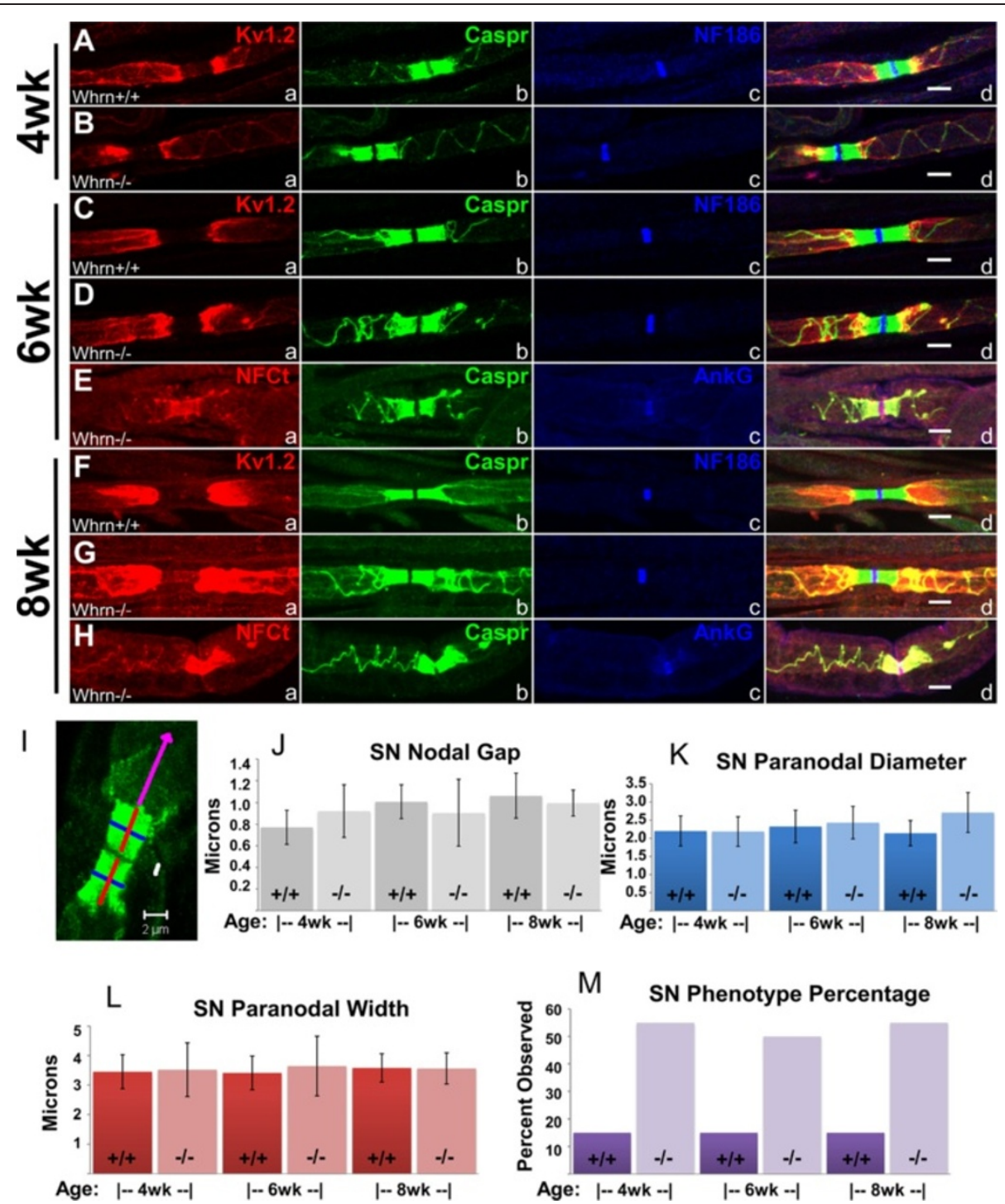

Figure 2 Loss of Whirlin in the peripheral nervous system results in disrupted paranodal compaction. A-H. 4, 6, 8-week-old teased sciatic nerve fibers either wild-type (Aa-Ad, Ca-Cd, Fa-Fd) or Whrn knockout (Ba-Bd, Da-Dd, Ea-Ed, Ga-Gd, Ha-Hd) immunostained against $\mathrm{K}_{\mathrm{v}} 1.2$ (Aa-Da, Fa, Ga, red), NFCt (Ea, Ha, red), Caspr (Ab-Hb, green), NF186 (Ac-Dc, Fc, Gc, blue), AnkG (Ec, Hc, blue), and merged images (Ad-Hd). In all Whrn mutant panels, Caspr (Bb, Bd; Db, Dd; Eb, Ed; Gb, Gd; Hb, Hd, green) and paranodal NF155 (NFCt) (Ea, Ed; Ha, Hd, red) fail to compact properly at the paranodes. Nodal NF186 or AnkG are not affected $(\mathbf{A} \mathbf{c}, \mathbf{d}-\mathbf{H} \mathbf{c}, \mathbf{d}$, blue). Scale bars $(\mathbf{A d}-\mathbf{H d})=5 \mu \mathrm{m}$. I. Sample image shows parameters of various domain measurements in (nodal gap in white, paranodal diameter in blue, paranodal width in red, and counting of springlike phenotype in purple) using 10 micron caliber, Caspr-immunostained wild-type and Whrn ${ }^{-/-}$fibers. J-L. No statistically significant differences were observed comparing 4, 6, and 8-week-old wild-type and mutant fibers with concern to nodal gap (J), paranodal diameter (K), or paranodal width (L) ( $\mathrm{N}=20$ for each genotype/age combination). Note the greater percentage of paranodes with compaction issues in mutant fibers (M, light purple bars) likely contributes to the increased deviation in paranodal widths (L, light red bars).

paranodal loops fails to occur in Whrn knockout mice at 4,6 , and 8 weeks of age.

During initial quantification we observed that larger diameter myelinated fibers had proportionally more paranode compaction defects compared to thinner caliber myelinated fibers, so we imaged and assembled twenty Caspr-stained confocal images for each genotype and time point $(4,6$, and 8 week-old) to assess any subtle, sub-micron paranodal changes by light microscopy due to Whrn loss. Next, we measured various dimensions of the paranode (Figure 2I). Note images collected were from $\sim 10$ micron caliber myelinated neurons. Using Zeiss software, micron measurements were recorded, tabulated, and reported as averages with standard deviations for the nodal gap (Figure 2J), the paranodal diameter (Figure $2 \mathrm{~K}$ ), paranodal width (Figure 2L), as well as the percentage of paranodes which display paranodal compaction abnormalities (Figure 2M). The 
nodal gap (Figure 2J) for wild-type and $\mathrm{Whrn}^{-1-}$ sciatic nerves was $0.77 \pm 0.16 \mu \mathrm{m}$ and $0.92 \pm 0.24 \mu \mathrm{m}$ at 4 weeks, $1.01 \pm 0.16 \mu \mathrm{m}$ and $0.91 \pm 0.31 \mu \mathrm{m}$ at 6 weeks, and $1.06 \pm 0.21 \mu \mathrm{m}$ and $1.00 \pm 0.12$ at 8 weeks respectively. No statistically significant difference was noted between ages or genotypes with respect to nodal gap. The paranodal diameter (Figure $2 \mathrm{~K}$ ) for wild-type and $\mathrm{Whrn}^{-/-}$sciatic nerves was $2.20 \pm 0.42 \mu \mathrm{m}$ and $2.19 \pm 0.41 \mu \mathrm{m}$ at 4 weeks, $2.33 \pm 0.45 \mu \mathrm{m}$ and $2.43 \pm 0.45 \mu \mathrm{m}$ at 6 weeks, and $2.14 \pm 0.35 \mu \mathrm{m}$ and $2.71 \pm 0.55$ at 8 weeks respectively. With respect to paranodal diameter, measurements were similar between all groups. The paranodal width (Figure 2L) for wild-type and $\mathrm{Whrn}^{-/-}$sciatic nerves was $3.45 \pm 0.58 \mu \mathrm{m}$ and $3.52 \pm 0.91 \mu \mathrm{m}$ at 4 weeks, $3.41 \pm 0.57 \mu \mathrm{m}$ and $3.64 \pm 1.01 \mu \mathrm{m}$ at 6 weeks, and $3.58 \pm 0.48 \mu \mathrm{m}$ and $3.56 \pm 0.53$ at 8 weeks respectively. No statistically significant difference was found in paranodal width. The paranodal compaction phenotype percentage (Figure 2M) for wild-type and $\mathrm{Whrn}^{-/-}$sciatic nerves was $15 \%(3 / 20)$ and $55 \%(11 / 20)$ at 4 weeks, $15 \%$ $(3 / 20)$ and $50 \%(10 / 20)$ at 6 weeks, and $15 \%(3 / 20)$ and $55 \%(11 / 20)$ respectively. As a reminder, the increase in the Whrn knockout phenotype percentage represents the shift in selection from all fibers $(1.8 \%)$ to larger-caliber myelinated fibers $(50 \%)$. Note the greater percentage of paranodes with compaction issues in $\mathrm{Whrn}^{-/-}$sciatic nerves (Figure 2M, light purple bars) likely contributes to the increased deviation in paranodal widths (Figure 2L, light red bars). Additionally, comparisons between 8 weekold wild-type and $\mathrm{Whrn}^{-1-}$ sciatic nerve fibers demonstrated no significant difference in the conduction velocity (average $\sim 30 \mathrm{~m} / \mathrm{s}$ ) or waveforms (data not shown) of measured compound action potentials in two separate measurement trials.

\section{Whrn knockout mouse sciatic nerve and spinal cord myelinated fibers display paranodal compaction abnormalities throughout development}

To expand on the characterization of Whrn loss with respect to myelinated domain organization, we examined a larger developmental window from postnatal ages 2 weeks to 1 year. Wild-type and Whrn knockout sciatic nerves revealed the following percentages of Caspr-stained phenotype-positive paranodes (Figure 2): $1.5 \%$ and $2.7 \%$ at 10 weeks, $0.9 \%$ and $1.8 \%$ at 20 weeks, $0.8 \%$ and $1.5 \%$ at 30 weeks, $0.5 \%$ and $1.4 \%$ at 40 weeks, and $0.7 \%$ and $1.5 \%$ at 1 year, respectively. Similar to the 4-8 week studies (Figure 2), we immunostained fibers with nodal, paranodal, and juxtaparanodal markers, as well as the axonal cytoskeletal protein markers $4.1 \mathrm{~B}$ and heavy chain Neurofilament (Nfl-H) given Whrn's known cytoskeletal scaffolding role. We observed no differences in nodal formation using NF186 (Figure 3Ac, Bc) in 2 week-old fibers. Abnormalities in paranodal formation and com- paction were observed in Caspr-stained $\mathrm{Whrn}^{-1-}$ fibers (Figure 3Bb, Bf) compared to wild-type (Figure 3Ab, Af). Overall $\mathrm{K}_{\mathrm{v}} 1.2$ juxtaparanodal signal appeared similar between 2 week-old wild-type (Figure 3Aa) and $\mathrm{Whrn}^{-/-}$ fibers (Figure 3Ba) with accumulation and enrichment of $\mathrm{K}_{\mathrm{v}} 1.2$ channels (Figure $3 \mathrm{Bd}$ ) neighboring Caspr within the internodal region. No obvious difference in intensity or localization of Nfl-H (Figure 3Ae, Be) or 4.1B (Figure $3 \mathrm{Ag}, \mathrm{Bg}$ ) was found in 2 week-old sciatic nerve fibers between Whrn genotypes. With 10 week-old sciatic nerve fibers, we observed no differences in nodal domains using NF186 (Figure 3Cc, Dc). Like 8 week-old fibers (Figure 2F-H), we observed 10 week-old $\mathrm{Whrn}^{-/-}$ fibers with abnormal paranodal compaction (Figure $3 \mathrm{Db}$, $\mathrm{Df}$ ), when compared to wild-type fibers (Figure $3 \mathrm{Cb}, \mathrm{Cf}$ ). Juxtaparanodal domains stained with $\mathrm{K}_{\mathrm{v}} 1.2$ (Figure 3Da vs. $3 \mathrm{Ca})$ appear more diffuse but similar in overall intensity in 10 week-old $\mathrm{Whrn}^{-/-}$fibers when compared to wildtype. We observed slight enrichment of Nfl-H (Figure 3Ce vs. $3 \mathrm{De}$ ) and $4.1 \mathrm{~B}$ (Figure $3 \mathrm{Cg}$ vs. $3 \mathrm{Dg}$ ) within the paranodal region of 10 week-old wild-type sciatic nerves compared to Whrn knockout mice, but the overall intensity of Nfl-H and 4.1B along the remaining axon appeared similar. Immunostaining of 20, 30, and 40 week-old wild-type sciatic nerve fibers were performed and domain organization was identical to 10 week-old wild-type fibers (Figure 3Ca-k). Looking at 20, 30, and 40 weekold $\mathrm{Whrn}^{-/-}$peripheral nerve fibers, we observed no differences in nodal domains between Whrn wild-types (Figure 3Cc) or knockout mice using NF186 (Figure 3EcGc). Similar to 10 week-old fibers (Figure 3Db, Df), we observed numerous 20, 30, and 40 week-old Whrn null fibers stained with Caspr (Figure 3Eb,d-Gb,d) with abnormal paranodal compaction when compared to wildtype fibers (Figure 3Cb,d). In 20, 30, and 40-week old $\mathrm{Whrn}^{-/-}$fibers, juxtaparanodal domains stained with $\mathrm{K}_{\mathrm{v}} 1.2$ (Figure 3Ea-Ga) showed numerous breaks at sites of Caspr signal, appeared less symmetrical, and displayed similar overall signal when compared with wildtype (Figure 3Ca). Cytoskeletal marker Nfl-H staining (Figure 3Ee-Ge) was generally uniform within the axon, and $4.1 \mathrm{~B}$ (Figure $3 \mathrm{Eg}-\mathrm{Gg}$ ) was present in all axonal domains except the node in 20,30, and 40 week-old $\mathrm{Whrn}^{-1-}$ sciatic nerves. One-year-old sciatic nerves showed no difference in nodal organization (Figure 3Hc vs. 3Ic). One year-old $\mathrm{Whrn}^{-1-}$ sciatic nerves (Figure 3Ib, d) revealed abnormal paranodal compaction compared to wild-type fibers (Figure $3 \mathrm{Hb}, \mathrm{d}$ ). Notably, we observed paranodal compaction defects in older $\mathrm{Whrn}^{-1-}$ sciatic nerve fibers (40 week-old and 1 year-old) like blocks and bulges in Caspr signal as well as the spring-like phenotype observed in younger fibers (4-30 week-old). As seen previously, the juxtaparanodal domains of $\mathrm{Whrn}^{-1-}$ peripheral nerves stained with $\mathrm{K}_{\mathrm{v}} 1.2$ (Figure 3Ia) 


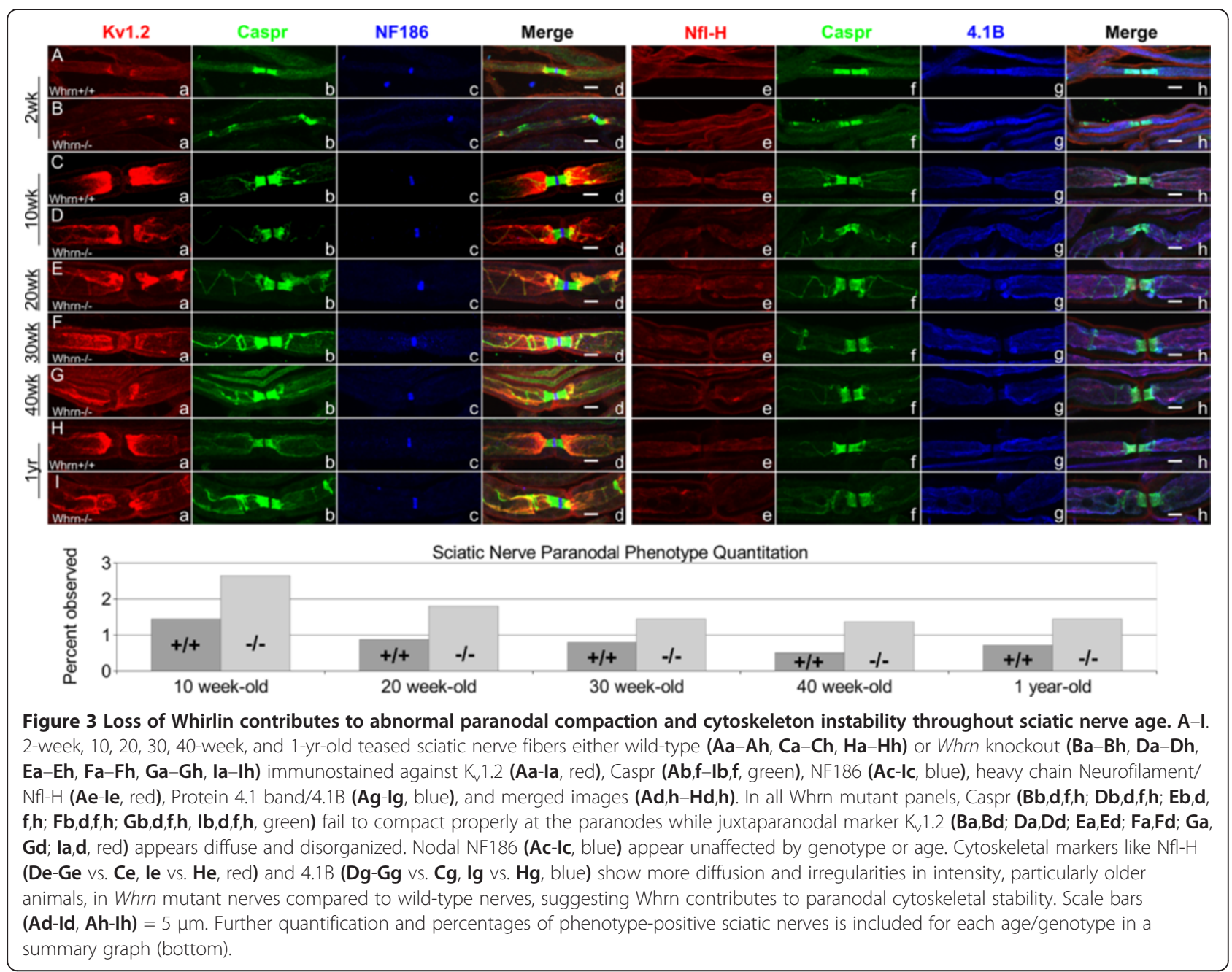

showed significant asymmetric enrichment and periodic breaks as well as overlap with Caspr signal when compared with one year-old wild-type fibers (Figure 3Ha). Also, patchy cytoskeletal-deficient staining was observed in older $\mathrm{Whrn}^{-/-}$fibers (Figure 3Gh, 3Ih) near the paranode. Cytoskeletal marker $\mathrm{Nfl}-\mathrm{H}$ staining was weakly enriched within the paranode of the wild-type axon (Figure 3Hh) and less uniform within $\mathrm{Whrn}^{-/}$fibers with paranodal compaction problems (Figure 3Ih). Likewise, 4.1B showed disrupted juxtaparanodal cytoskeletal staining in one year-old $\mathrm{Whrn}^{-1-}$ fibers (Figure 3Ig) which was not observed in the wild-type fibers (Figure $3 \mathrm{Hg}$ ).

We also sought to examine the effects of Whrn loss on axonal domain organization in the central nervous system (CNS). Utilizing white matter tracts in the spinal cord, we were able to identify by immunostaining subtle but consistent differences in paranodal compaction. As in the PNS (Figure 3), we stained longitudinal spinal cord sections with nodal, paranodal, and juxtaparanodal markers (Figure 4). Wild-type and Whrn knockout spinal cord fibers revealed the following percentages of Casprstained phenotype-positive paranodes (Figure 3): 0.6\% and $1.7 \%$ at 10 weeks, $1.2 \%$ and $3.4 \%$ at 20 weeks, $1.3 \%$ and $2.5 \%$ at 30 weeks, and $1.0 \%$ and $2.4 \%$ at 40 weeks. No differences in nodal organization were observed (Figure 3.4Ac vs. 3.4Bc) in 2 week-old spinal cord sections. At 10, 20, 30, 40 week-old, nodal organization appeared similar between wild-type and $\mathrm{Whrn}^{-/-}$spinal cord fibers using NF186 (Figure 4Cc vs. 4Dc, 4Ec vs. $4 \mathrm{Fc}, 4 \mathrm{Gc}$ vs. $4 \mathrm{Hc}, 4 \mathrm{Ic}$ vs. $4 \mathrm{Jc}$ ). Immunostaining with paranodal Caspr revealed subtle and infrequent paranodal abnormalities in 2 week-old Whrn null spinal cord fibers (Figure $4 \mathrm{Bb}$ ) compared to wild-type (Figure 4Ab). In contrast, obvious and regular paranodal compaction defects were observed in 10 (Figure $4 \mathrm{Cb}$ vs. $4 \mathrm{Db}$ ), 20 (Figure $4 \mathrm{~Eb}$ vs. $4 \mathrm{Fb}$ ), 30 (Figure $4 \mathrm{~Gb}$ vs. $4 \mathrm{Hb}$ ), and 40 week-old (Figure 4Ib vs. 4Jb) $\mathrm{Whrn}^{-1-}$ spinal cords compared to their wild-type controls. Finally, juxtaparanodal $\mathrm{K}_{\mathrm{v}} 1.2$ immunostaining of 10 (Figure $4 \mathrm{Ca}$ vs. 4Da), 20 (Figure 4Ea vs. 4Fa), 30 (Figure 4Ga vs. 4Ha), and 

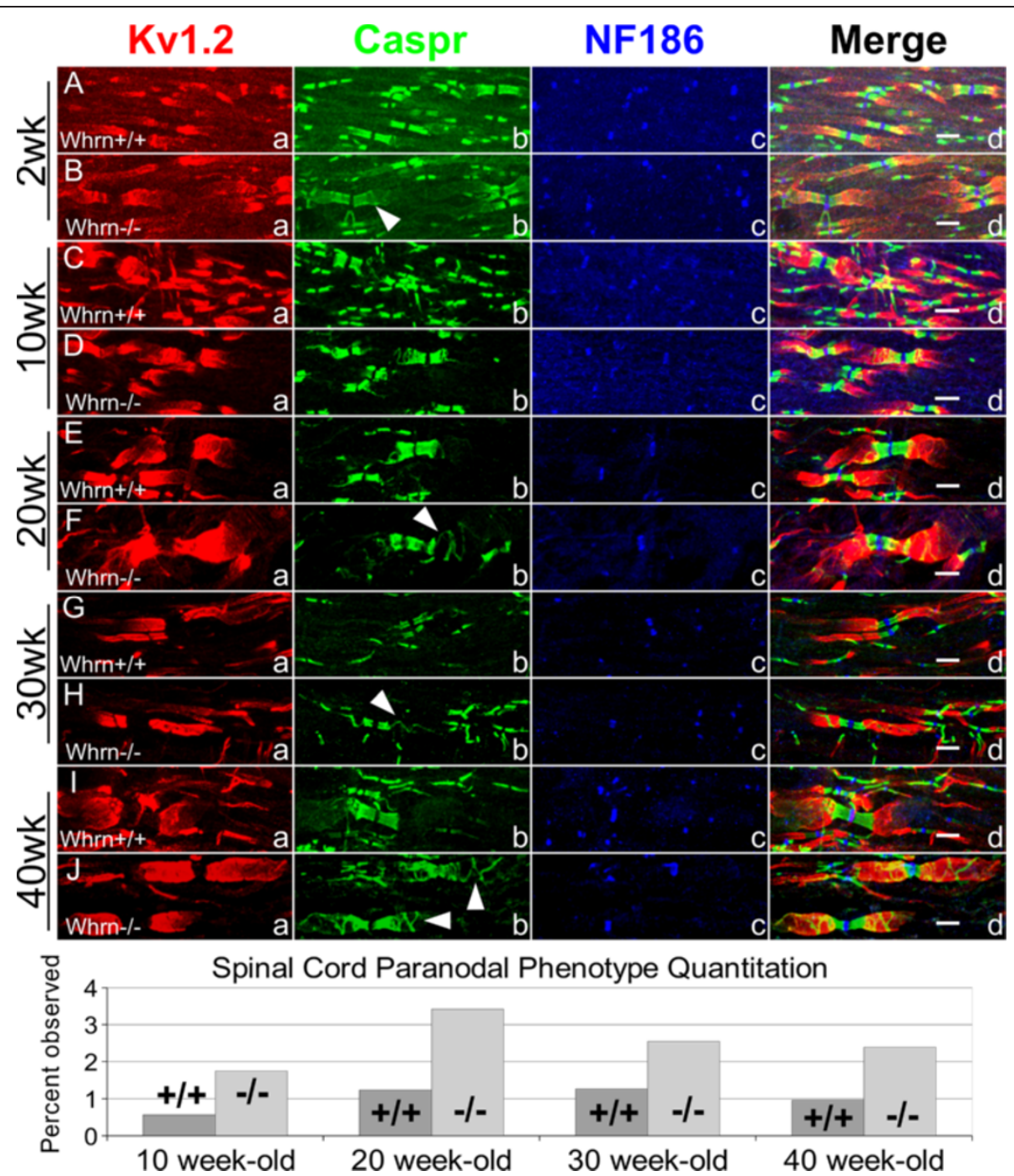

Figure 4 Loss of Whirlin contributes to abnormal paranodal compaction throughout development in the spinal cord. A-J. 2-week, 10, 20, 30, 40-week teased sciatic nerve fibers either wild-type (Aa-Ad, Ca-Cd, Ea-Ed, Ga-Gd, la-Id) or Whrn knockout (Ba-Bd, Da-Dd, Fa-Fd, Ha-Hd, Ja-Jd) immunostained against Caspr (Ac-Jc, green), Kv1.2 (Aa-Ja, red), NF186 (Ac-Jc, blue), and merged images (Ad-Jd). Nodal NF186 (Ac-Jc, blue) appear unaffected by age or genotype. In Whrn mutant spinal cords, Caspr (Bb,d; $\mathbf{b}, \mathbf{d} ; \mathbf{F b}, \mathbf{d} ; \mathbf{H b}, \mathbf{d} ; \mathbf{J b}, \mathbf{d} ;$ green) does not compact properly at the paranodes compared to wild-type Caspr (Ab,d; Cb,d; Eb,d; Gb,d; $\mathbf{l b}, \mathbf{d}$; green). In addition, juxtaparanodal marker $K_{v} 1.2$ (Ba,d; Da,d; $\mathbf{F a}, \mathbf{d} ; \mathbf{H a}, \mathbf{d} ; \mathbf{J a}, \mathbf{d} ;$ red) in Whrn mutant spinal cords shows similar signal intensity and occasional colocalization with Caspr-stained myelin loops. Scale bars $(\mathbf{A d}-\mathbf{J d})=5 \mu \mathrm{m}$. Further quantification and percentages of phenotype-positive spinal cord fibers is included for each age/genotype in a summary graph (bottom).

40 week-old (Figure 4Ia vs. 4Ja) wild-type spinal cord myelinated axons showed similar overall expression as Whrn knockout axons, but consistent juxtaparanodal disorganization and colocalization with loosened Casprstained myelin loops was observed most often in $\mathrm{Whrn}^{-/-}$ fibers. In summary, there is significant evidence that loss of Whrn disrupts normal paranodal compaction in 1040 week-old myelinated spinal cord axons with subsequent effects on juxtaparanodal organization. Taken together, both the peripheral and central nervous systems likely utilize the cytoskeletal properties of Whrn to help stabilize the cellular organization between myelinating glial cells and neurons around the paranodal region throughout development.

Whirlin knockout mice have cerebellar Purkinje cells with bead-like, axonal swellings

To determine the effects of Whrn loss on cerebellar Purkinje cell morphology, we immunostained cerebellar slices from 6 week-old wild-type, Whrn knockout, and double Whrn and 4.1B [14] null animals (Figure 5). Given that Caspr [9] and CGT [28,29], two genes critical for formation of a proper paranode, display Purkinje axonal swellings and cytoskeletal disorganization, we 


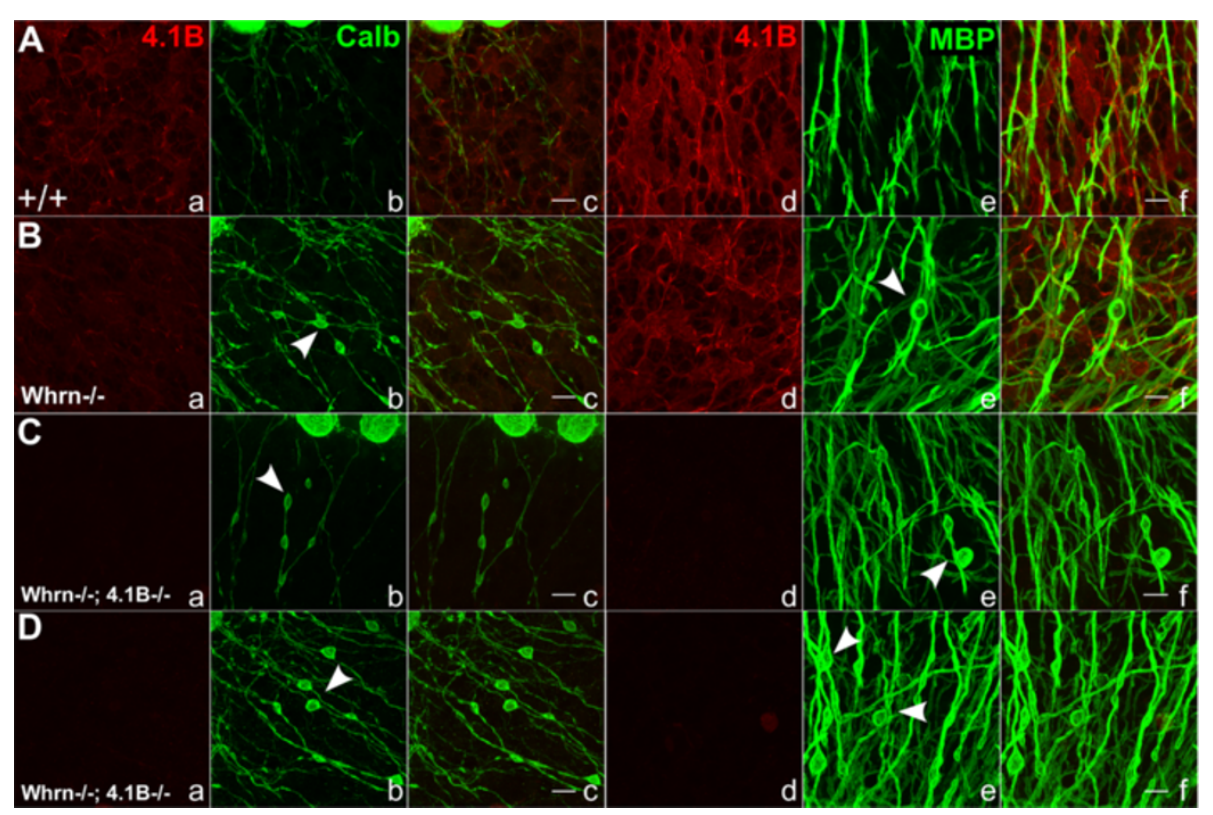

Figure 5 Whirlin alone and combination 4.1B/Whirlin knockout mice have cerebellar Purkinje cells with bead-like, axonal swellings. A-D. 6-week sagittal sections of mouse cerebellum in either wild-type (Aa-Af), Whrn knockout (Ba-Bf), or double Whrn knockout and 4.1B null (Ca-Cf, Da-Df) immunostained against Protein 4.1B band/4.1B (Aa-Da, Ad-Dd, red), Calbindin (Ab-Db, green), myelin basic protein/MBP $\mathbf{v A e - D e}$, green), and merged images (Ac,f-Dc,f). No striking difference was observed between wild-type and Whrn knockout slices in 4.1B staining (Aa,d vs. Ba,d) and only non-specific staining was present in 4.1B null animals (Ca-Da, Cd-Dd). In Whrn mutant cerebellum sections (Ba-Bf), Purkinje axonal swellings appear bead-like using both Calb (Bb,c vs. Ab,c) and MBP (Be,f vs. Ae,f) in comparison to a uniform, thin caliber axon in wild-type fibers (Aa-f). Additionally, secondary ablation of 4.1B protein resulted in a greater number of swellings observed with Calb $(\mathbf{C b}, \mathbf{c}-\mathbf{D b}, \mathbf{c}$ vs. Bb,c) and MBP (Ce,f-De,f vs. Be,f) when compared with Whrn knockouts alone suggesting these cytoskeletal elements help prevent cytoskeletal disorganization in Purkinje cell axons. Scale bars (Ac-Dc, Af-Df) $=10 \mu \mathrm{m}$.

were curious as to the effects of the combined loss of Whrn and 4.1B. While no obvious differences in localization was observed using our Whrn antibody (data not shown) in any of these genotypes, we immunostained against 4.1B (Figure 5Aa,d-Da, d; red), Purkinje-specific Calbindin (Figure 5Ab-Db, green), and glial-specific myelin basic protein/MBP (Figure $5 \mathrm{Ae}-\mathrm{De}$, green). There was no difference in $4.1 \mathrm{~B}$ staining intensity (Figure 5Aa, d vs. 5Ba,d) between wild-type and Whrn knockout slices. Upon examining $\mathrm{Whrn}^{-/-}$cerebellum sections (Figure 5Ba-Bf), Purkinje axons appeared to contain bead-like, swellings along their extensions through the granular layer using both Calb (Figure $5 \mathrm{Bb}, \mathrm{c}$ vs. $5 \mathrm{Ab}, \mathrm{c}$, white arrowheads) and MBP (Figure 5Be,f vs. 5Ae,f, white arrowheads) in comparison to a uniform caliber axon in wild-type fibers (Figure $5 \mathrm{Ab}, \mathrm{c}, \mathrm{e}, \mathrm{f}$ ). The secondary loss of $4.1 \mathrm{~B}$ protein resulted in more swellings observed with Calb (Figure $5 \mathrm{Cb}, \mathrm{c}-5 \mathrm{Db}, \mathrm{c}$ vs. $5 \mathrm{Bb}, \mathrm{c}$, white arrowheads) and MBP (Figure $5 \mathrm{Ce}, \mathrm{f}-5 \mathrm{De}, \mathrm{f}$ vs. 5Be,f, white arrowheads) when compared with Whrn null animals alone. In summary, the cytoskeletal elements Whrn and 4.1B likely have an assistive role in preventing cytoskeletal accumulation and disorganization within cerebellar Purkinje cell axons in a similar phenotypic manner to Caspr null mice [9].
Ultrastructural abnormalities in Whrn knockout sciatic nerve, spinal cord fibers, and cerebellar Purkinje axons To further understand Whrn's role in myelinated axons, transmission electron microscopy was performed in order to examine the ultrastructural architecture in myelinated axons in the PNS (Figure 6) and CNS (Figure 7) of 7 week-old and 3 month-old wild-type and $\mathrm{Whrn}^{-/-}$ mice. Low-magnification, cross-section electron micrographs of wild-type (Figure 6A) and Whrn knockout (Figure 6B, C) myelinated sciatic nerve fibers showed the typical organization of tightly bound, electrondense myelin wraps around the internodal region of the axonal membrane. Accumulation of mitochondria and lipid vesicles (Figure $6 \mathrm{~B}, \mathrm{C}$ vs. $6 \mathrm{~A}$ and $6 \mathrm{E}, \mathrm{F}$ vs. $6 \mathrm{D}$, flat arrowheads) in the internodal regions was clearly observed in Whrn knockout animals compared to wild-type sciatic nerve fibers. Given the potential role of the mesaxon in the observed light microscope phenotype [15], we found no striking differences in the ultrastructural organization or arrangements of the inner mesaxon along the internodal region at 7 weeks or 3 months of age (data not shown). Consistent with our immunostaining data, no obvious differences in nodal organization were observed in either genotype (Figure 6G vs 6H,I). Higher magnification along the wild-type paranodal region (Figure 6), 

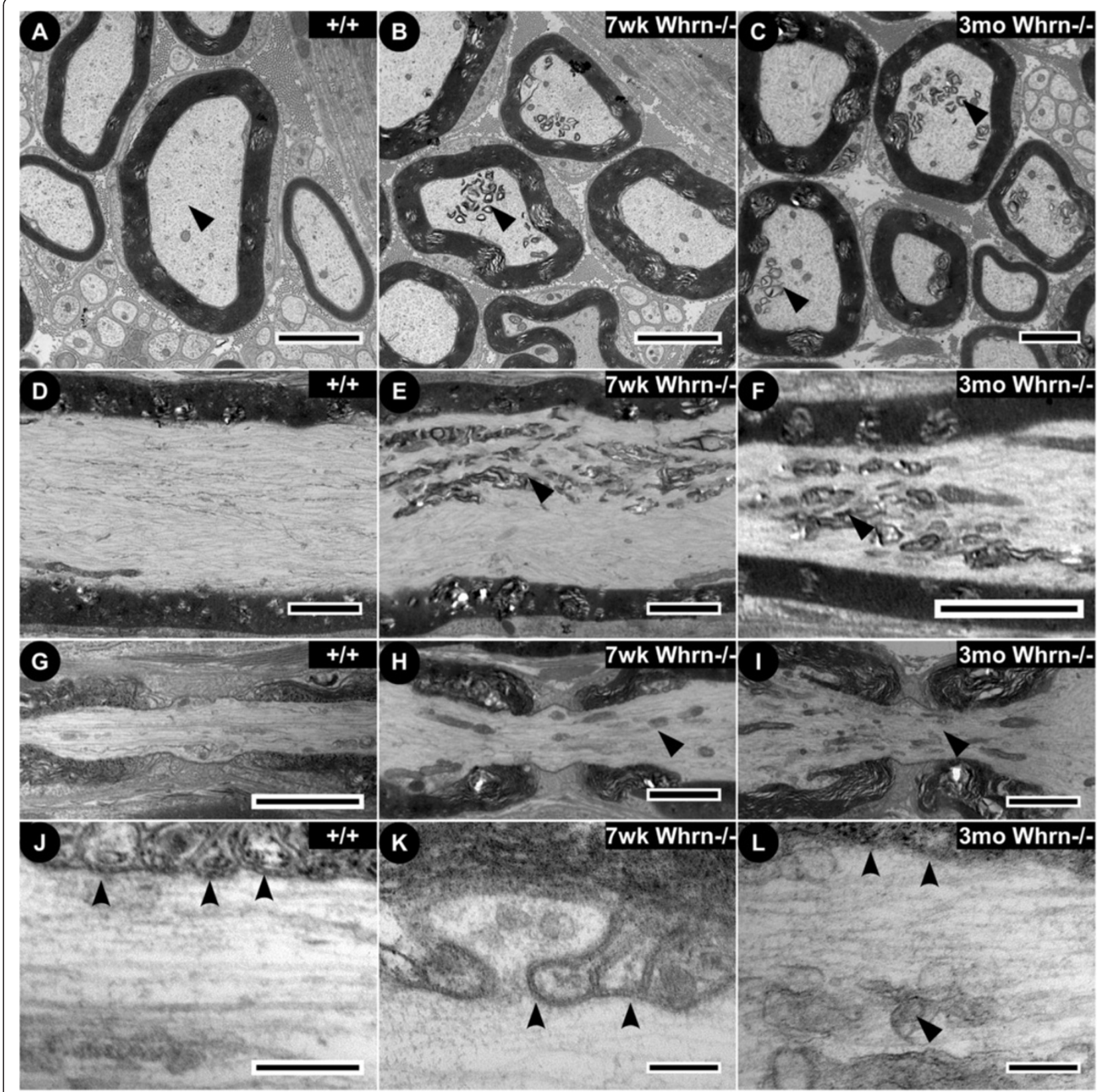

Figure 6 Ultrastructural examination of Whirlin knockout sciatic nerves reveals organelle accumulation and cytoskeletal disruption. Low magnification electron micrographs through the internodal regions of sciatic nerves in wild-type (7 week-old) and Whrn knockout mice (7 week-old, 3 month-old) in cross section (A vs. B, C) and longitudinal orientations (D vs. E, F). Overall cellular organization between Whrn knockout and wild-type sciatic nerve fibers is conserved with tightly compacted myelin around each axon. Low magnification, longitudinal electron micrographs through the nodal and paranodal regions of sciatic nerves are presented for wild-type (7 week-old, G) and Whrn knockout mice (7 week-old, $\mathbf{H} ; 3$ month-old, I). At a higher magnification, the wild-type (J) paranodal loops have clearly defined characteristic transverse, electron-dense septa (concave arrowheads) and parallel arrays of cytoskeletal elements. In contrast, Whrn mutant paranodal septa (K, L, concave arrowheads) are less definitive and fuzzy with associated accumulation of organelles (flat arrowheads), particularly mitochondria and transport vesicles. Scale bars: A-I, 2 $\mu \mathrm{m}, \mathbf{J}-\mathbf{L}, 400 \mathrm{~nm}$.

concave arrowheads) revealed the hallmark electrondense AGSJs formed between the glial paranodal loops and axolemma and accompanying parallel arrays of cytoskeletal elements in the axon. In contrast, the Whrn knockout paranodal region of 7 week-old (Figure 6K) and
3 month-old (Figure 6L) displayed poorly defined but present AGSJs (Figure 6K,L, concave arrowheads), less organized neurofilaments and microtubules, and consistent accumulation of mitochondria and lipid vesicles (Figure 6H, 6I, 6L, flat arrowheads) in the paranodal 


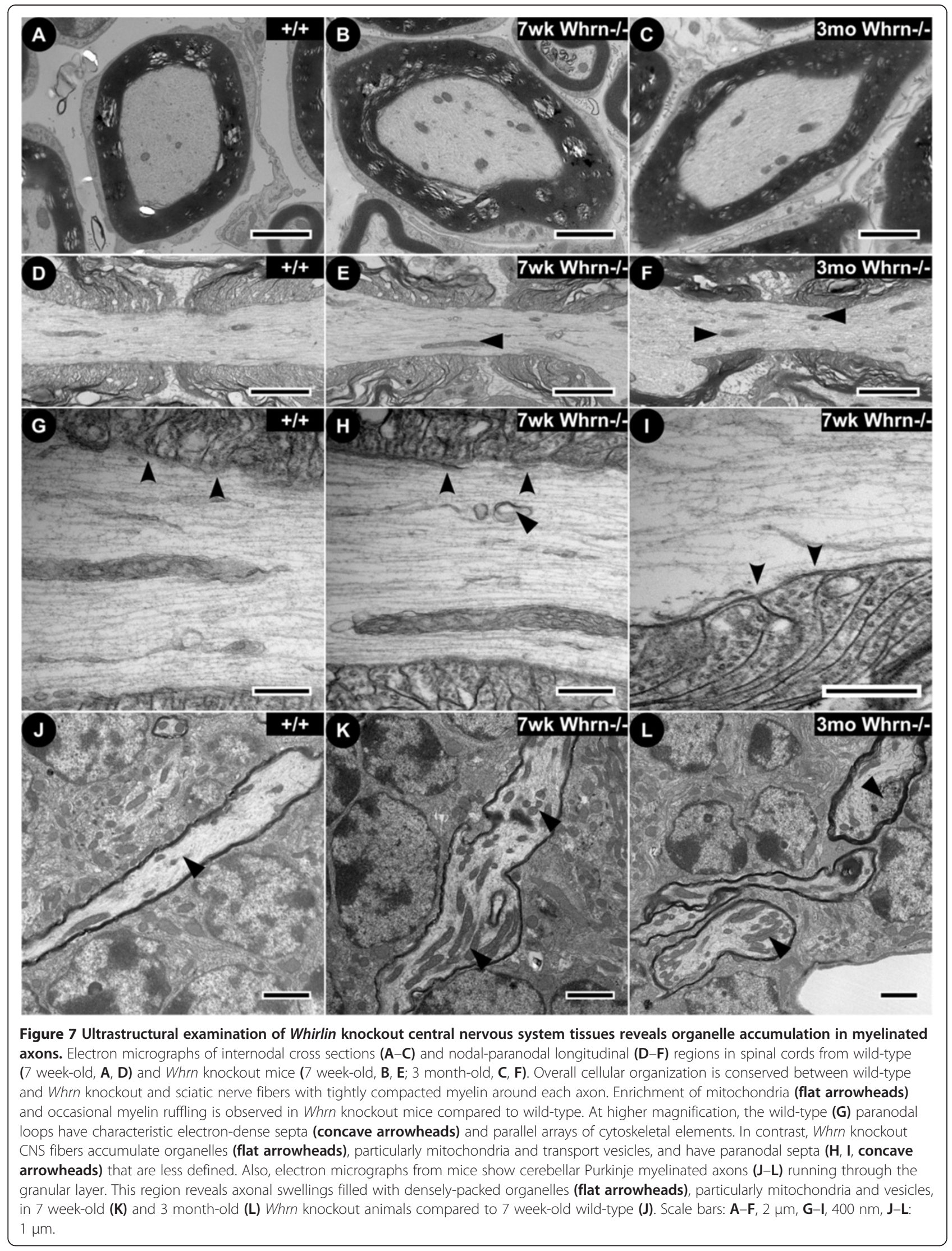


region. The ultrastructural phenotypes displayed at both 7 weeks and 3 months of age suggest that Whrn is important for the long-term maintenance and the overall structure of the myelinated axons.

In the central nervous system, low-magnification, electron micrographs of wild-type (Figure 7A) and Whrn knockout (Figure 7B, C) myelinated fibers showed slight differences in spinal cord cross-sections. Like the peripheral nerves, the overall organization between glial cell and neuron remained similar between wild-type and $\mathrm{Whrn}^{-/-}$fibers. However, mitochondria were slightly more abundant in Whrn knockout fibers. No obvious ultrastructural differences were observed in the node of either genotype (Figure 7D-F), but greater accumulation of mitochondria (flat arrowheads) and lipid vesicles was observed in the paranodal and juxtaparanodal regions. Higher magnification along the paranodal region (Figure 7G-I, concave arrowheads) revealed the expected electron-dense AGSJs formed between myelin loops and the axolemma. Like in the PNS, the Whrn knockout paranodal region of 7 week-old (Figure $7 \mathrm{H}$ ) and 3 month-old (Figure 7I) displayed poorly defined but present AGSJs (concave arrowheads) and accumulation of mitochondria and lipid vesicles (Figure $7 \mathrm{H}$, flat arrowhead) in the paranodal region. Examining the cerebellum, we observed Purkinje axon swellings in Whrn knockout fibers (Figure 7K-L). Low-magnification electron micrographs of 7 week-old wild-type (Figure 7J) and Whrn knockout at 7 weeks (Figure 7K) and 3 months (Figure 7L) of age showed striking differences in Purkinje axon myelinated fibers in the cerebellum granular layer. This region shows axonal swellings filled with densely-packed organelles, particularly mitochondria and vesicles (Figure 7K-L, flat arrowheads). Taken together, the ultrastructural analyses of Whrn knockout mice demonstrate that Whrn is critical for the stability of paranodal organization, proper axonal cytoskeletal arrangements, and prevention of sub-cellular organelle accumulation in myelinated axons.

\section{Conclusions}

Cellular and molecular interactions between neurons and glia establish and stratify the numerous tasks of the nervous system. In particular, linkage of cellular membranes with the underlying cytoskeleton via cytoskeletal linker proteins helps maintain the specialized cellular arrangements necessary to glial and neuronal function. The potential of Whrn to link plasma membrane proteins with multiple cytoskeletal protein partners has been well established [16,17,19-24], yet a precise role for Whrn in the central or peripheral nervous system or even more specifically in myelinated neurons has not been examined. In myelinated axons, axonal membrane proteins like Caspr and Caspr2 help stabilize domain organization through linkage of $4.1 \mathrm{~B}$ to the underlying cytoskeleton.
This loss of organization at both the light and electron microscopy level is readily apparent in mutant mice lacking Caspr [7], Caspr2 [12], and 4.1B [14]. Here we report that Whrn knockout animals reveal defective cytoskeletal organization and accumulation of organelles in the myelinated fibers. Our phenotypic analyses of Whrn knockout mice demonstrate that loss of Whrn disrupts proper paranodal compaction and long-term stability of the myelinated axons throughout development.

\section{Whrn alternative splicing and phenotypes}

To understand the function of Whrn in the nervous system, one must be considerate of Whrn mRNA splice variants and rule out confounding mouse genetic strain differences. Currently, two mutant mouse strains exist for Whirlin: the whirler (Whrn ${ }^{w i / w i}$ ) mouse has a spontaneous genomic deletion of exons 6-9 while the Whrn $\left(\mathrm{Whrn}^{-/-}\right)$knockout mouse [17] has a targeted exon 1 deletion. We examined the localization of several myelinated axon markers (Caspr, 4.1B, $\mathrm{K}_{\mathrm{v}} 1.2$, and NF186) in Whrn wild-type mice from each background strain and observed no disruptions in the localization of these proteins or the morphology of the paranodes (data not shown). Our initial studies comparing $\mathrm{Whrn}^{-/-}$to $\mathrm{Whrn}^{\text {wi/wi }}$ mouse strains suggested myelinated domain organization, early paranodal disorganization, and nerve conduction $(\sim 30 \mathrm{~m} / \mathrm{s})$ was indistinguishable between each mutant mouse line. Given the novelty of the phenotype, we additionally substantiated no mouse strain effects after back-crossing to isogenic C57BL6 mice for two generations. Given the consistency of the mutant phenotype regardless of background mouse genetics, we were assured the phenotype was attributed to loss of Whrn function and not alternative Whrn splice variants or mouse genetic background variation.

\section{Scaffolding by Whrn and previously established protein networks may underlie paranodal compaction and stabilization}

Whrn has several established roles derived from its complex and numerous interactions with other protein partners within the ear and eye. This complexity comes from identifying and comparing the human and mouse Whrn mutants and splice variants. The significant bulk of Whrn research has been performed in the ear and eye since human WHRN mutations contribute to a subset of Usher syndromes. In the eye, full-length Whrn colocalizes with the transmembrane proteins Usherin and VLGR1 at the periciliary membrane complex in photoreceptors [17]. Studies demonstrate the two Nterminal PDZ domains of the full-length Whrn isoform are responsible for this interaction. In the ear, the shorter Whrn isoform has a more significant role since mutation and/or loss of Whrn's C-terminus correlates 
to poorer hearing deficits compared to Whrn's N-terminus. The Whrn short isoform interacts through its Prolinerich domain and last PDZ domain with Myosin XVa and $\mathrm{Mpp} 1 / \mathrm{p} 55$. Additional reports demonstrate Whrn protein expression in the mouse cerebrum, cerebellum, and brainstem [25], rat cerebellum [27], and in the Drosophila central nervous system [26]. Taken together, Whrn has a well-documented history of shared proteinprotein interaction across multiple model systems and cells.

We propose that Whrn as a cytoskeletal scaffold crosslinks a subcellular axonal meshwork to stabilize the paranode, juxtaparanode, or both, and that in the absence of Whrn, subcellular compaction of the paranode and organization of underlying microtubules, neurofilaments, cause cytoskeletal disorganization leading to an accumulation of mitochondria, and lipid vesicles along myelinated axons. Here we propose the potential sites of interaction for Whrn within these regions given its established protein-protein interactions in vivo. Within the paranode, the intracellular c-terminus of Caspr contains a SH3 domain, a potential site of interaction with Whrn's Proline-rich domain. The paranode and juxtaparanode are also enriched for $4.1 \mathrm{~B}$, a known protein partner in the ear stereocilia for Mpp1/p55 which interacts with Whrn [22]. Caspr2 also contains a PDZbinding motif which could potentially interact with one of Whrn's PDZ domains [12]. Finally, the c-terminus of Whrn has the potential for self-oligomerization [20], allowing for even more complex networks of proteinprotein interaction within the myelinated axon.

Domain organization in myelinated axons is a complicated developmental process, culminating from intrinsic and extrinsic cellular factors. Here we demonstrate that Whrn expression is important for proper axonal domain organization and expand the role of Whrn outside the ear and eye. The phenotypes observed in the myelinated axons highlight that the paranodal-juxtaparanodal interface represents a substantial region for insight into paranodal stabilization and potential interaction with the axonal cytoskeleton. In light of recent evidence of 4.1G's role at the internode and mesaxon, this paranodal-juxtaparanodal interface may represent an important subdomain in the study of myelinated fibers. Whrn's protein domains have the potential to stabilize the paranodal myelin loops and associated cytoskeleton through direct or indirect interactions with Caspr, 4.1B, or other unidentified cytoskeletal proteins. These observations are correlated using several techniques including biochemistry, light and electron microscopy. Our observed paranodal phenotypes are consistent throughout development ( 2 wk-1 yr) and similar between central and peripheral nervous systems. One final important consideration about cytoskeletal linker proteins, both Whrn and $4.1 B$ null mice have no statistical difference in conduction velocities in sciatic nerves compared to wild-type mice, despite having clear paranodal instability in Caspr-stained myelinated fibers. Such data suggest cytoskeletal linker proteins may be functionally redundant with respect to myelinated domain organization and may require secondary or tertiary genetic ablations to achieve any measurable electrophysiological effects. To this point, we observed the increase in Purkinje axonal swellings in the double Whrn; $4.1 B$ null mouse cerebellum compared to the single Whrn knockout or wild-type mouse cerebellum. In conclusion, our observations indicate Whrn acts as a cytoskeletal scaffolding protein that is essential for proper paranodal compaction and stabilization of the axonal cytoskeleton for long-term health of myelinated axons.

\section{Methods}

Animals

Whrn exon 1 homozygous mutants used were obtained from Dr. Jun Yang's lab (University of Utah, Salt Lake City, Utah, 84132). The mice were backcrossed to C57BL6 mice (JAX Laboratories \#000664, Bar Harbor, Maine) for two generations and maintained as heterozygous $\mathrm{Whrn}^{+/-}$breeding stocks. All animal experiments were performed according to Institutional Animal Care and Use Committee approved guidelines for ethical treatment of laboratory animals at the University of North Carolina at Chapel Hill and the University of Texas Health Science Center at San Antonio.

\section{Genotyping}

Genomic DNA from mouse tails and/or toe snips was extracted using a kit according to manufacturer's instructions (Sigma-Aldrich Extract-N-Amp ${ }^{\mathrm{TM}}$ Tissue PCR Kit (XNAT2)). Cycling conditions used were: $95^{\circ} \mathrm{C}$ for $5 \mathrm{~min}, 35$ cycles of $\left(95^{\circ} \mathrm{C}\right.$ for $30 \mathrm{sec}, 68^{\circ} \mathrm{C}$ for $\left.1.5 \mathrm{~min}\right)$, and $68^{\circ} \mathrm{C}$ for $3 \mathrm{~min}$. Primer sequences for PCR were obtained from Dr. Jun Yang's lab as follows: common WhrnRP (Pdzg5r) CAGGGAAGTTGAGGCACACGG, wild-type Whrn+FP (pdzg1) GGGTGAGTGAATGCCA GCCAG, and knockout Whrn-FP (PNT3A) GAGATCA GCAGCCTCTGTTCCAC. The Whrn+ product size is $894 \mathrm{bp}$; the Whrn-product size is $700 \mathrm{bp}$.

\section{Generation of Whrn antibody}

We generated rabbit, guinea pig, and rat polyclonal antiWhrn antibodies similar to previous literature [23]. A full length Whrn mouse cDNA construct in pcDNA3.1 was obtained from Dr. Jun Yang's lab. Regions encoding amino-acid residues 220-326 and 699-804 of mouse Whirlin (Genbank: NP_001008791.1) were subcloned individually into pGEX4T1 and expressed in Escherichia coli (BL21; Stratagene, La Jolla, CA). Fusion proteins were isolated by incubating with Glutathione Sepharose 4 Fast Flow (GE Healthcare, Sweden). Each fusion 
protein was used to immunize a rabbit, guinea pig, or rat (Cocalico Biologicals Inc, Reamstown, PA). cDNAs encoding amino acids 220-326 or 699-804 of mouse Whrn were also introduced into pMAL-c2x (New England Biolabs, Beverely, MA), transformed into E. coli (DE3 BL21; Stratagene) and induced to express the corresponding maltose binding (MBP) fusion protein. The expressed MBP-fusion proteins were purified using amylose resin (New England Biolabs, Beverely, MA) and then linked to a NHS-activated Sepharose 4 Fast Flow (GE Healthcare, Sweden). Only antisera from the immunized rabbit \#349 (RbWhrn349) was affinity purified using the corresponding MBP-Whrn fusion protein.

\section{RNA preparation and RT-PCR}

Sciatic nerves, dorsal root ganglia, and spinal cord sections were removed from p21 Whrn mutant and wild-type mice. Tissue was stored and processed in RNAlater Stabilization Reagent (QIAGEN). Total RNA was isolated using QIA shredder columns (QIAGEN) and RNeasy Mini Kit (QIAGEN). RNA concentration was estimated and approx. 5 ng RNA was used for reverse transcription followed by PCR amplification using the MyTaq One-Step RT-PCR kit (Bioline). RT-PCR analysis was performed on three separate sets of animals. Cycling conditions used were: $45^{\circ} \mathrm{C}$ for $20 \mathrm{~min}, 95^{\circ} \mathrm{C}$ for $1 \mathrm{~min}, 35$ cycles of $\left(95^{\circ} \mathrm{C}\right.$ for $10 \mathrm{sec}, 62^{\circ} \mathrm{C}$ for $10 \mathrm{sec}, 72^{\circ} \mathrm{C}$ for $\left.30 \mathrm{sec}\right)$, and $72^{\circ} \mathrm{C}$ for $5 \mathrm{~min}$. Primers used for PCR were as follows: Actin (Ex2) FP: GCTCCGGCATGTGCAA, Actin (Ex4) RP: A GGATCTTCATGAGGTAGT. Whrn (Ex1) FP: ACCA GATTCTGCGCGTCAAC, Whrn (SalI-Ex4) RP: tccgG TCGACcacctccagaatctggtctc; Whrn (EcoRI-Ex9) FP: CC CAgaattcGGGGCCTGCCTTCCACC, Whrn (SalI-Ex10) RP: cgggGTCGACgttggcacctccgcgg.

\section{Other antibodies and immunostaining reagents}

The following antisera were previously described: guinea pig and rabbit anti-Caspr [7,10,30], guinea pig antiNF186 and guinea pig anti-pan Neurofascin [10,30], guinea pig anti-4.1B antibodies [14], and mouse antiCalbindin [31]. Other primary antibodies used include the following: mouse anti- $\mathrm{K}_{\mathrm{v}} 1.2$ (University of California Davis/NIH NeuroMab Facility; K14/16), mouse antiCASK (University of California Davis/NIH NeuroMab Facility; K56A/50), mouse anti-Ankyrin G (University of California Davis/NIH NeuroMab Facility; N106/36), mouse anti-Neurofilament $\mathrm{H}$ (Chemicon, MAB1623), rabbit anti-alpha-Tubulin (Cell Signaling \#2144) and anti-Myelin Basic Protein (Abcam, SMI-94). Secondary antibodies used for immunofluorescence were Alexa Fluor-488, -568, and -647 conjugated (Invitrogen). HRPconjugated secondary antibodies were purchased from Jackson ImmunoResearch.

\section{Immunostaining}

Briefly, sciatic nerves were removed from anesthetized littermate wild-type and Whrn mutants of either sex and fixed in 4\% paraformaldehyde in PBS for 15-30 min. The nerves were washed with PBS three times (10 min each) and stored at $4^{\circ} \mathrm{C}$ until teased. The nerves were teased into individual fibers in PBS, mounted on glass slides, and dried overnight at room temperature. Fibers were either immediately used for immunostaining or stored at $-80^{\circ} \mathrm{C}$ until needed. Teased nerve slides were submerged in acetone (methanol instead for anti-MBP staining) at $-20^{\circ} \mathrm{C}$ for $20 \mathrm{~min}$ then washed with PBS, followed by immunostaining [4]. For spinal cord sections and cerebellar sections, wild-type and mutants were deeply anesthetized and intra-cardially perfused with PBS followed by ice-cold 4\% paraformaldehyde in PBS. The spinal cord or cerebellum was dissected out and post-fixed in $4 \%$ paraformaldehyde overnight at $4^{\circ} \mathrm{C}$. The tissues were rinsed with PBS and sectioned to $30 \mathrm{um}$ using a Vibratome (Leica). The sections were then immediately immunostained as previously described $[9,10]$. Primary antibodies for immunostaining were used at the following concentrations overnight at $4^{\circ} \mathrm{C}$ : RbCaspr @1:500, GPNF186@1:400, MsIgG2b- $\mathrm{K}_{\mathrm{v}} 1.2$ @1:200, GPNFCt @1:400, GP-beta-IVspectrin @1:1000, GP4.1B @1:10000, and MsIgG-Nfl-H @1:1000, MsIgG1-Calb @1:1000, and MsIgG1-MBP @1:200.

\section{Immunoblotting}

Sciatic nerves and dorsal root ganglia from littermate wild-type and mutants of either sex were excised and processed using a glass homogenizer in ice-cold lysis buffer $(50 \mathrm{mM}$ Tris-HCl, pH 7.5, $150 \mathrm{mM} \mathrm{NaCl}$, $10 \mathrm{mM}$ EDTA, 1\% Triton X-100, 1\% SDS, and a protease mixture tablet). The lysate was incubated for $30 \mathrm{~min}$ on ice and then centrifuged at $16,000 \times \mathrm{g}$ for $20 \mathrm{~min}$ at $4^{\circ} \mathrm{C}$. The sciatic nerve or dorsal root ganglia supernatant was saved for further processing. Spinal cords from littermate wild-type and mutants of either sex were excised and either directly processed or frozen at $-80^{\circ} \mathrm{C}$. Spinal cords were homogenized using a glass mortar and pestle on ice with lysis buffer (50 mM Tris- $\mathrm{HCl}, \mathrm{pH} 7.5$, $150 \mathrm{mM} \mathrm{NaCl}, 10 \mathrm{mM}$ EDTA, 1\% Triton X-100, and a protease inhibitor mixture tablet) and incubated for $30 \mathrm{~min}$ on ice with occasional trituration. The homogenate was centrifuged at $1000 \mathrm{xg}$ for $10 \mathrm{~min}$ at $4^{\circ} \mathrm{C}$. The supernatant was collected and subjected to an additional centrifugation at $100,000 \times \mathrm{g}$ for $30 \mathrm{~min}$ at $4^{\circ} \mathrm{C}$. The resulting second supernatant was collected and saved for further processing. Protein concentrations of final lysates were determined using the Lowry assay (BC assay; BioRad). Lysates were resolved by SDS-PAGE and transferred onto nitrocellulose membranes, followed by immunoblotting procedures described previously [4]. Primary 
antibodies for immunoblotting were used at the following concentrations for $1 \mathrm{hr}$ at room temperature: Affinitypurified RbWhrn349 @ 1:1000 (overnight at $4^{\circ} \mathrm{C}$ ), GP Caspr @ 1:2000, GPNFCt @ 1:2000, Rb4.1B@ 1:50000, Ms IgG1-CASK@1:50000, RbCaspr2@ 1:50000, RbTubulin @ 1:2000 (overnight at $4^{\circ} \mathrm{C}$ ).

\section{Electrophysiology}

The conduction velocity measurements of sciatic nerves were carried out on three separate wild-type $(+/+)$ and $\mathrm{Whrn}^{-/-}$mice as described previously $[10,30]$.

\section{Image analysis and software}

Confocal images were captured with a Zeiss LSM510 microscope. Scanning parameters were optimized for wild-type tissues and maintained for scanning the mutant tissues. Immunofluorescence images for sciatic nerves and spinal cords are composite projections from Z stacks of three to six sections (0.6um scan step) or stacks of ten to twenty sections (0.6um scan step) for cerebellar slices. Software used for assembling figures included Zeiss LSM Image Browser (v4.2), Image (v1.47d), GIMP (v2.82), and OpenOffice (v3.4.1).

\section{Quantification of phenotype and statistics}

For the initial quantification, we utilized a blinded counting strategy to best estimate the spring-like phenotype. Teased sciatic nerves were prepared from wild-type or $\mathrm{Whrn}^{-/-}$mice. One individual prepared all teased slides and randomly assigned a number to each slide. Once completed, the individual compiled a table of genotypes matched to assigned numbers. Blinded to that table, a second individual immunostained the numbered slides and counted wild-type and $\mathrm{Whrn}^{-/-}$Caspr-stained paranodes. Immunostained paranodes were counted under a fluorescent microscope at 40× magnification. Any paranode with 3 or more spring-like, loops were considered phenotypepositive. Data was compiled as the percentage of phenotype-positive paranodes out of total paranodes. Phenotype percentages per slide were matched to genotype and then a final average phenotype percent for wild-type or $\mathrm{Whrn}^{-1-}$ fibers was calculated as well the standard error of the mean (SEM). A standard t-test was used to calculate the statistical significance (p-value) between the percent for 7 week-old wild-type or $\mathrm{Whrn}^{-/-}$fibers. Similar phenotype quantitation was applied to 10,20 , 30 , and 40 wk old sciatic nerve and spinal cords. All measured data was tabulated in Microsoft Excel. A pvalue of 0.05 was considered to indicate a significant difference between groups.

For secondary quantitation of paranodal parameters in 4-8 week-old animals, Caspr-stained paranodes were imaged using a confocal LSM510 microscope and accompanying Zeiss Image software. A composite projection of
Z-stacks was performed for each image. Large-caliber sciatic nerve images ( 10um total diameter glial-edge to glial edge) were analyzed given the initial observation of more phenotype-positive paranodes in large vs. small caliber axons. Twenty wild-type and twenty $\mathrm{Whrn}^{-/-}$ paranode images were selected for each time point (60 total images). Using Zeiss software tools, each paranode was measured in microns for nodal gap (distance between nodal-paranodal boundaries in white), paranodal diameter (distance across axon caliber in blue), paranodal width (distance from nodal-paranodal boundary to paranodaljuxtaparanodal boundary in red), and phenotype percentage (a paranode was considered phenotype-positive if the purple line from the paranodal-juxtaparanodal boundary crossed a Caspr AGSJ line three times (i.e. 1.5 circular myelin wrapping loops) and phenotype-negative if less than three times). All measured data was tabulated in Microsoft Excel. The average and standard deviation of each age and genotype was graphed for nodal gap, paranodal diameter, and paranodal width. Phenotype percentage was reported as a percent of phenotypepositive paranodes out of twenty total counted for that age and genotype.

\section{Transmission electron microscopy}

Animal tissues were fixed using 4\% Formaldehyde/1\% Glutaraldehyde (4CF1G) via intra-cardiac perfusion for 30 minutes. Tissues were dissected out and placed in $4 \mathrm{CF} 1 \mathrm{G}$ to post-fix overnight at $4^{\circ} \mathrm{C}$. Tissues were then processed by UTHSCSA Electron Microscopy core. Core processing steps as follows: (1) buffer rinse in $0.1 \mathrm{M}$ phosphate buffer for 5 minutes to overnight, (2) postfixation in $1 \%$ Zetterqvist's buffered Osmium Tetroxide for 30 minutes, (3) buffer rinse in Zetterqvist's buffer for 3 minutes, (4) en bloc staining in $2 \%$ aqueous uranly acetate for 20 minutes, (5) dehydration in $70 \%$ alcohol for 10 minutes, then $95 \%$ alcohol for 10 minutes, then $100 \%$ alcohol twice for 10 minutes, then propylene oxide twice for 10 minutes, (5) resin infiltration in 1:1 propylene oxide:resin for 30 minutes then $100 \%$ resin for 30 minutes under 25 psi vacuum. Once embedded, tissue was sliced in $90 \mathrm{~nm}$ sections and placed on copper grids. Grids were stained with uranyl acetate for 30 seconds in the microwave and then with Reynold's lead for 20 seconds. Samples were imaged at $80 \mathrm{kV}$ on a JEOL 1230 electron microscope using AMT (advanced microscopy techniques) software.

\footnotetext{
Abbreviations

AGSJ: Axo-glial septate junction; Whrn: Whirlin; Caspr: Contactin-associated protein; 4.1B: Protein 4.1B (brain) band.
}

Competing interests

The authors declared that they have no competing interests. 


\section{Authors' contributions}

JG performed genotyping and phenotype quantitation, immunostaining and confocal imaging; JY provided Whrn exon 1 knockout mouse strain; MG and BK provided whirler mouse strain and various Whrn-related reagents; JG and MB participated in the design of the study, assembled figures, and drafting of the manuscript. All authors approved the final manuscript.

\section{Acknowledgements}

We are grateful to Thomas Friedman for generously sharing antibodies and reagents. We thank Alaine Pribisko for conducting the electrophysiology on sciatic nerves. We thank Barbara Hunter in the UTHSCSA electron microscopy core for her help in preparing the tissues for TEM. We thank Courtney Thaxton for comments on the manuscript and helpful discussions. We thank Vladimir Ghukasyan (UNC at Chapel Hill, NC) and Exing Wang (UTHSCSA, San Antonio, TX) for help with microscopy. The confocal microscopy cores were supported by Grants: NIH-NCI P30 CA54174 (San Antonio Cancer Institute), NIH-NIA P30 AG013319 (Nathan Shock Center) and (NIH-NIA P01AG19316). This work was supported by NIH grants GM63074 (MAB), GM063074-07S1 (JRG/MAB), and UNC-CH Medical Scientist Training Program Grant T32 GM008719 (JRG)

\section{Author details}

${ }^{1}$ Department of Cell and Molecular Physiology, University of North Carolina School of Medicine, Chapel Hill, NC 27599, USA. ²Ophthalmology and Visual Sciences, Moran Eye Center and Neurobiology and Anatomy, University of Utah, Salt Lake City, UT 84132, USA. 'aboratory of Cell Structure and Dynamics, National Institute on Deafness and Other Communication Disorders, National Institutes of Health, Bethesda, MD 20892, USA. ${ }^{4}$ Department of Physiology, University of Texas School of Medicine, Health Science Center, 7703 Floyd Curl Drive, San Antonio, TX 78229, USA.

Received: 19 July 2013 Accepted: 3 September 2013

Published: 6 September 2013

\section{References}

1. Bhat MA: Molecular organization of axo-glial junctions. Curr Opin Neurobiol 2003, 13(5):552-559.

2. Salzer JL: Polarized domains of myelinated axons. Neuron 2003, 40(2):297-318.

3. Thaxton C, Bhat MA: Myelination and regional domain differentiation of the axon. Results Probl Cell Differ 2009, 48:1-28.

4. Thaxton C, Pillai AM, Pribisko AL, Dupree JL, Bhat MA: Nodes of Ranvier act as barriers to restrict invasion of flanking paranodal domains in myelinated axons. Neuron 2011, 69(2):244-257.

5. Rosenbluth J: Role of glial cells in the differentiation and function of myelinated axons. Int J Dev Neurosci 1988, 6(1):3-24.

6. Boyle ME, Berglund EO, Murai KK, Weber L, Peles E, Ranscht B: Contactin orchestrates assembly of the septate-like junctions at the paranode in myelinated peripheral nerve. Neuron 2001, 30(2):385-397.

7. Bhat MA, Rios JC, Lu Y, Garcia-Fresco GP, Ching W, St Martin M, Li J, Einheber S, Chesler M, Rosenbluth J, et al: Axon-glia interactions and the domain organization of myelinated axons requires neurexin IV/Caspr/ Paranodin. Neuron 2001, 30(2):369-383.

8. Tait S, Gunn-Moore F, Collinson JM, Huang J, Lubetzki C, Pedraza L, Sherman DL, Colman DR, Brophy PJ: An oligodendrocyte cell adhesion molecule at the site of assembly of the paranodal axo-glial junction. J Cell Biol 2000, 150(3):657-666.

9. Garcia-Fresco GP, Sousa AD, Pillai AM, Moy SS, Crawley JN, Tessarollo L, Dupree $J$, Bhat MA: Disruption of axo-glial junctions causes cytoskeletal disorganization and degeneration of Purkinje neuron axons. Proc Natl Acad Sci U S A 2006, 103(13):5137-5142.

10. Pillai AM, Thaxton C, Pribisko AL, Cheng JG, Dupree JL, Bhat MA: Spatiotemporal ablation of myelinating glia-specific neurofascin (NfasC NF155) in mice reveals gradual loss of paranodal axoglial junctions and concomitant disorganization of axonal domains. J Neurosci Res 2009, 87(8):1773-1793.

11. Zonta B, Tait S, Melrose S, Anderson H, Harroch S, Higginson J, Sherman DL, Brophy PJ: Glial and neuronal isoforms of Neurofascin have distinct roles in the assembly of nodes of Ranvier in the central nervous system. $J$ Cell Biol 2008, 181(7):1169-1177.
12. Poliak $S$, Salomon $D$, Elhanany $H$, Sabanay $H$, Kiernan $B$, Pevny L, Stewart $C L$, Xu X, Chiu SY, Shrager P, et al: Juxtaparanodal clustering of Shaker-like K+ channels in myelinated axons depends on Caspr2 and TAG-1. J Cell Biol 2003, 162(6):1149-1160.

13. Horresh I, Bar V, Kissil JL, Peles E: Organization of myelinated axons by Caspr and Caspr2 requires the cytoskeletal adapter protein 4.1B. J Neurosci: the official journal of the Society for Neuroscience 2010, 30(7):2480-2489.

14. Buttermore ED, Dupree JL, Cheng J, An X, Tessarollo L, Bhat MA: The cytoskeletal adaptor protein band $4.1 \mathrm{~B}$ is required for the maintenance of paranodal axoglial septate junctions in myelinated axons. J Neurosci: the official journal of the Society for Neuroscience 2011, 31(22):8013-8024

15. Ivanovic A, Horresh I, Golan N, Spiegel I, Sabanay H, Frechter S, Ohno S, Terada N, Mobius W, Rosenbluth J, et al: The cytoskeletal adapter protein 4.1G organizes the internodes in peripheral myelinated nerves. $J$ Cell Biol 2012, 196(3):337-344.

16. Mburu P, Mustapha M, Varela A, Weil D, El-Amraoui A, Holme RH, Rump A, Hardisty RE, Blanchard S, Coimbra RS, et al: Defects in whirlin, a PDZ domain molecule involved in stereocilia elongation, cause deafness in the whirler mouse and families with DFNB31. Nature genetics 2003, 34 (4):421-428.

17. Yang J, Liu X, Zhao Y, Adamian M, Pawlyk B, Sun X, McMillan DR, Liberman MC, Li T: Ablation of whirlin long isoform disrupts the USH2 protein complex and causes vision and hearing loss. PLOS genetics 2010, 6(5):e1000955.

18. Friedman TB, Schultz JM, Ahmed ZM, Tsilou ET, Brewer CC: Usher syndrome: hearing loss with vision loss. Adv Oto-Rhino-Laryng 2011, 70:56-65.

19. van Wijk E, van der Zwaag B, Peters T, Zimmermann U, Te Brinke H, Kersten FF, Marker T, Aller E, Hoefsloot LH, Cremers CW, et al: The DFNB31 gene product whirlin connects to the Usher protein network in the cochlea and retina by direct association with USH2A and VLGR1. Hum Mol Genet 2006, 15(5):751-765.

20. Delprat B, Michel V, Goodyear R, Yamasaki Y, Michalski N, El-Amraoui A, Perfettini I, Legrain P, Richardson G, Hardelin JP, et al: Myosin XVa and whirlin, two deafness gene products required for hair bundle growth, are located at the stereocilia tips and interact directly. Hum Mol Genet 2005, 14(3):401-410.

21. Okumura K, Mochizuki E, Yokohama M, Yamakawa H, Shitara H, Mburu P, Yonekawa H, Brown SD, Kikkawa Y: Protein 4.1 expression in the developing hair cells of the mouse inner ear. Brain Res 2010, 1307:53-62.

22. Mburu P, Kikkawa Y, Townsend S, Romero R, Yonekawa H, Brown SD: Whirlin complexes with $\mathrm{p} 55$ at the stereocilia tip during hair cell development. Proc Natl Acad Sci U S A 2006, 103(29):10973-10978.

23. Belyantseva IA, Boger ET, Naz S, Frolenkov Gl, Sellers JR, Ahmed ZM, Griffith AJ, Friedman TB: Myosin-XVa is required for tip localization of whirlin and differential elongation of hair-cell stereocilia. Nat Cell Biol 2005, 7(2):148-156.

24. Manor U, Disanza A, Grati M, Andrade L, Lin H, Di Fiore PP, Scita G, Kachar B: Regulation of stereocilia length by myosin XVa and whirlin depends on the actin-regulatory protein Eps8. Curr Biol : CB 2011, 21(2):167-172.

25. Wang L, Zou J, Shen Z, Song E, Yang J: Whirlin interacts with espin and modulates its actin-regulatory function: an insight into the mechanism of Usher syndrome type II. Hum Mol Genet 2012, 21(3):692-710.

26. Jepson JE, Shahidullah M, Lamaze A, Peterson D, Pan H, Koh K: Dyschronic, a Drosophila homolog of a deaf-blindness gene, regulates circadian output and Slowpoke channels. PLoS genetics 2012, 8(4):e1002671.

27. Yap CC, Liang F, Yamazaki Y, Muto Y, Kishida H, Hayashida T, Hashikawa T, Yano R: CIP98, a novel PDZ domain protein, is expressed in the central nervous system and interacts with calmodulin-dependent serine kinase. JNeurochem 2003, 85(1):123-134.

28. Dupree JL, Girault JA, Popko B: Axo-glial interactions regulate the localization of axonal paranodal proteins. J Cell Biol 1999, 147(6):1145-1152.

29. Marcus J, Dupree JL, Popko B: Myelin-associated glycoprotein and myelin galactolipids stabilize developing axo-glial interactions. J Cell Biol 2002, 156(3):567-577.

30. Thaxton C, Pillai AM, Pribisko AL, Labasque M, Dupree JL, Faivre-Sarrailh C, Bhat MA: In vivo deletion of immunoglobulin domains 5 and 6 in neurofascin (Nfasc) reveals domain-specific requirements in myelinated 
axons. J Neurosci: the official journal of the Society for Neuroscience 2010, 30(14):4868-4876

31. Buttermore ED, Piochon C, Wallace ML, Philpot BD, Hansel C, Bhat MA: Pinceau organization in the cerebellum requires distinct functions of neurofascin in Purkinje and basket neurons during postnatal development. J Neurosci: the official journal of the Society for Neuroscience 2012, 32(14):4724-4742.

doi:10.1186/1471-2202-14-96

Cite this article as: Green et al:: Whirlin, a cytoskeletal scaffolding protein, stabilizes the paranodal region and axonal cytoskeleton in myelinated axons. BMC Neuroscience 2013 14:96.

\section{Submit your next manuscript to BioMed Central and take full advantage of:}

- Convenient online submission

- Thorough peer review

- No space constraints or color figure charges

- Immediate publication on acceptance

- Inclusion in PubMed, CAS, Scopus and Google Scholar

- Research which is freely available for redistribution 